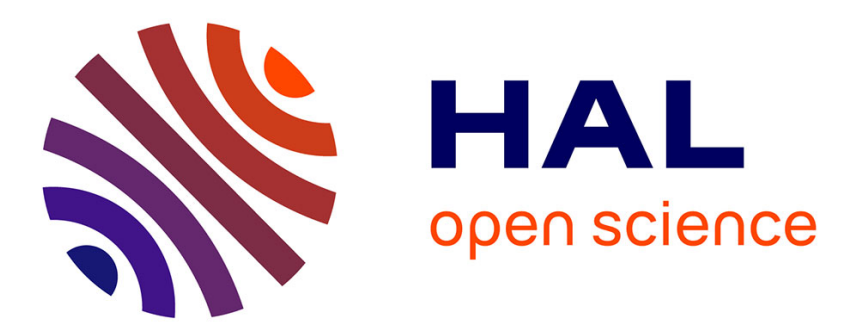

\title{
Laser control of ultracold molecule formation: The case of $\mathrm{RbSr}$
}

\author{
Adrien Devolder, Michèle Desouter-Lecomte, Osman Atabek, Eliane \\ Luc-Koenig, Olivier Dulieu
}

\section{- To cite this version:}

Adrien Devolder, Michèle Desouter-Lecomte, Osman Atabek, Eliane Luc-Koenig, Olivier Dulieu. Laser control of ultracold molecule formation: The case of RbSr. Physical Review A, 2021, 103 (3), 10.1103/PhysRevA.103.033301 . hal-03157026

\section{HAL Id: hal-03157026 \\ https://cnrs.hal.science/hal-03157026}

Submitted on 21 Nov 2021

HAL is a multi-disciplinary open access archive for the deposit and dissemination of scientific research documents, whether they are published or not. The documents may come from teaching and research institutions in France or abroad, or from public or private research centers.
L'archive ouverte pluridisciplinaire $\mathbf{H A L}$, est destinée au dépôt et à la diffusion de documents scientifiques de niveau recherche, publiés ou non, émanant des établissements d'enseignement et de recherche français ou étrangers, des laboratoires publics ou privés. 


\title{
Laser control of ultracold molecule formation: The case of $\mathrm{RbSr}$
}

\author{
Adrien Devolder* \\ Chemical Physics Theory Group, Department of Chemistry, and Center for Quantum Information and Quantum Control, \\ University of Toronto, Toronto, Ontario, Canada M5S $3 \mathrm{H6}$ \\ Michèle Desouter-Lecomte [i] \\ Université Paris-Saclay, CNRS, Institut de Chimie Physique, Bâtiment 349, 91405 Orsay Cedex, France \\ Osman Atabek (1) \\ Université Paris-Saclay, CNRS, Institut des Sciences Moléculaires d'Orsay, Bâtiment 520, 91405 Orsay Cedex, France \\ Eliane Luc-Koenig and Olivier Dulieu 10 \\ Université Paris-Saclay, CNRS, Laboratoire Aimé Cotton, Bâtiment 505, 91405 Orsay Cedex, France
}

(Received 25 November 2020; accepted 10 February 2021; published 2 March 2021)

\begin{abstract}
We have studied the formation of ultracold RbSr molecules with laser pulses. After discussing the advantages of the Mott insulator phase for the control with pulses, we present two classes of strategies. The first class involves two electronic states. Two extensions of stimulated Raman adiabatic passage (STIRAP) for multilevel transitions are used: alternating STIRAP (A-STIRAP) and straddle STIRAP (S-STIRAP). Both transfer dynamics are modeled and compared. The second class of strategies involves only the electronic ground state and uses infrared (IR) and terahertz (THz) pulses. The molecular bond is first created by the application of a $\mathrm{THz}$ chirped pulse or $\pi$-pulse. Subsequently, the molecules are transferred to their rovibrational ground level using IR pulses. For this last step, different optimized pulse sequences that were obtained through optimal control techniques have been studied. The relative merits of these strategies in terms of efficiency and robustness are discussed with respect to the experimental feasibility based on present laser technologies.
\end{abstract}

DOI: 10.1103/PhysRevA.103.033301

\section{INTRODUCTION}

Ultracold molecules are promising systems for a large number of applications, including quantum simulation [1-5], quantum computation [6-15], ultracold chemistry [16,17], and precision measurements [18-22]. Therefore, their formation remains an important objective. Ultracold alkali-alkali molecules were created first For that purpose, the most commonly used method is magnetoassociation [23,24] based on a magnetic Feshbach resonance (MFR) [25-27]. After their formation the molecules occupy a loosely bound level, not suitable for the above-cited applications. A transfer to the absolute ground level is necessary, and it is achieved via a stimulated Raman adiabatic passage (STIRAP) [28,29]. Magnetoassociation still represents a challenge for the formation of ultracold molecules containing a closed-shell atom, such as alkaline-earth or ytterbium [30]. This is precisely the case of $\mathrm{RbSr}$, which is a promising candidate for building a quantum simulator of lattice spin systems [2], one of the main Hamiltonian models in condensed matter physics. RbSr molecules could also be interesting for precision measurements, thanks to ultranarrow doublet-quartet spin-forbidden transitions. Re-

\footnotetext{
*Corresponding author: adrien.devolder@utoronto.ca
}

cently, narrow MFRs for RbSr were observed [31], but they have not yet been exploited for magnetoassociation.

An alternative to magnetic-field-based methods is the formation using lasers. Actually, the first ultracold molecules were created with continuous-wave $(\mathrm{cw})$ lasers via photoassociation [32]. The efficiency of molecule formation by photoassociation is limited, however, by atom losses, induced by spontaneous emission. For this reason, photoassociation has been replaced by magnetoassociation for the formation of alkali-alkali molecules. The above-mentioned limitations of magnetoassociation when addressing closed-shell atoms offer a new perspective for laser methods, especially if spontaneous emission can be avoided, which can hardly be done when relying on cw lasers. Furthermore, even without spontaneous emission, cw lasers induce Rabi oscillations. For an efficient formation, a time dependence must be introduced through the use of pulsed lasers. It is worth noting that a time dependence is also present in magnetoassociation, allowing the adiabatic passage.

In this paper, we consider two different strategies (Fig. 1). The first is based on STIRAP, which was already used for the formation of ultracold $\mathrm{Sr}_{2}$ molecules [33,34], and it implies excited electronic states during the transfer [Fig. 1(a)]. More particularly, advanced derivatives of STIRAP were considered, namely A-STIRAP and S-STIRAP [35-37]. The second strategy addresses only the electronic ground state via the 

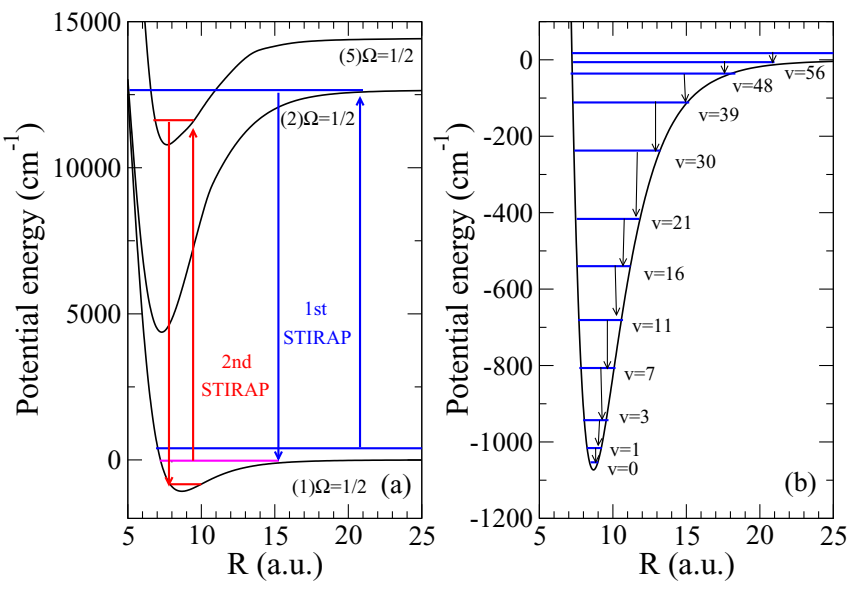

FIG. 1. Two strategies for creating RbSr molecules in their absolute ground level, either via two STIRAP transfers (left panel), or using $\mathrm{THz}$ and infrared pulses inducing rovibrational transitions inside the electronic ground state. In the left panel, the levels involved in the first STIRAP are drawn in blue, while the ones involved in the second STIRAP are drawn in red. The intermediate level, involved in both schemes, is drawn in magenta. In the right panel, the vibrational levels involved in the transfer are drawn as blue lines. The frequencies of the transitions of the second strategy (right panel) are given in Table III.

use of infrared and terahertz lasers [Fig. 1(b)], as previously suggested by Kotochigova [38] and Juarros et al. [39] relying on cw lasers.

The structure of the paper is as follows. In Sec. II, we discuss the issues related to the use of pulsed lasers in the formation of molecules and how we can circumvent them by trapping the atomic pairs in a Mott insulator. In Sec. III, the related Hamiltonian is presented. The formation of molecules following the first strategy via STIRAP methods is discussed in Sec. IV. Section V is devoted to the second strategy involving a single electronic state. Finally, in Sec. VI we discuss the pros and cons of the two strategies in relation to current laser technologies.

\section{FORMATION OF ULTRACOLD MOLECULES WITH PULSED LASERS}

At least two main issues must be solved when attempting the formation of a chemical bond with laser pulses. First, pulses only interact with colliding atom pairs. Unfortunately, from an ultracold nondegenerate atomic gas, this involves only a fraction of atoms. At first glance, for the conversion of all atom pairs to ultracold molecules, a solution would be the consecutive applications of several similar pulses. However, as shown with picosecond pulses [40] or repeating STIRAP, the application of pulses can also dissociate the previously formed molecules, unless control strategies aiming at the formation of very long-lived Feshbach resonances (zero-width resonances) are specifically addressed [41]. Thus, the repetition of the pulse sequences would not improve the efficiency of the molecular formation due to photodissociation. Secondly, the colliding pairs do not occupy a single continuum translational state, but a continuous distribution of states. As the coherent control with laser pulses is a unitary process and cannot reduce the entropy, the occupation of a single state at the end of the process is unlikely.

These difficulties can be overcome by trapping ultracold atoms in an optical lattice. A Bose-Einstein condensate (BEC) of ultracold atoms is prepared and adiabatically loaded into a three-dimensional (3D) optical lattice by increasing the lattice potential. During the adiabatic loading, the system undergoes a superfluid-to-Mott insulator phase transition [42]. Aiming at the formation of homonuclear diatomic molecules, a Mott insulator (MI) with two atoms per site is the starting point $[43,44]$, while for heteronuclear diatomic molecules the starting point is a double MI with many sites occupied by one atom of each species [45-51].

The trapping in MI can solve the two above-cited issues. First, the pulse sequence can be applied to all pairs at the same time and independently. Therefore, merely a single pulse sequence is sufficient. Secondly, the trapping induces the quantization of relative translation motions. If the entropy is low enough, most atom pairs in the MI occupy the lowest vibrational level of their lattice well. The control of a scattering process now implies the transfer between discretized levels, for which some efficient methods exist, such as the adiabatic passage with a chirped pulse, the $\pi$-pulse, or the STIRAP [52].

An alternative to the trapping in MI is the use of optical tweezers. In this case, one pair of atoms is trapped by optical tweezers and we can control the formation of a single molecule [53,54]. In the following, we focus on the MI approach as the starting point, all discussed strategies, however, presenting the potential to be transposed to the case of trapping by optical tweezers.

\section{HAMILTONIAN OF A TRAPPED ATOMIC PAIR}

\section{A. Separation of the center of mass and the relative motion}

The following situation is under consideration: two MI with, respectively, an ${ }^{87} \mathrm{Rb}$ atom and an ${ }^{84} \mathrm{Sr}$ atom per site are overlapped. At each site, the $\mathrm{Rb}(\mathrm{Sr})$ atoms of mass $m_{\mathrm{Rb}}\left(m_{\mathrm{Sr}}\right)$ feel a harmonic potential of frequency $\omega_{\mathrm{Rb}}\left(\omega_{\mathrm{Sr}}\right)$. The total Hamiltonian depending on the center-of-mass position $\vec{R}_{\mathrm{CM}}$ and the interatomic distance $\vec{R}$ of the atom pair is given by [55]

$$
\begin{aligned}
\hat{H}_{\text {trap }}= & -\frac{\hbar^{2}}{2 M} \nabla_{\vec{R}_{\mathrm{CM}}}^{2}+\frac{1}{2} M \omega_{\mathrm{CM}}^{2} R_{\mathrm{CM}}^{2}-\frac{\hbar^{2}}{2 \mu} \nabla_{R}^{2}+\hat{H}_{\text {rot }} \\
& +\hat{H}_{\mathrm{el}}+\hat{H}_{\mathrm{SO}}+\frac{1}{2} \mu \omega_{\mathrm{rel}}^{2} R^{2}+\mu \Delta \omega^{2} \vec{R}_{\mathrm{CM}} \cdot \vec{R} .
\end{aligned}
$$

The first term corresponds to the kinetic energy of the center of mass with total mass $M$. The second term is the harmonic potential felt by the center of mass in the trap with the frequency $\omega_{\mathrm{CM}}=\sqrt{\frac{m_{\mathrm{Rb}} \omega_{\mathrm{Rb}}^{2}+m_{\mathrm{Sr}} \omega_{\mathrm{Sr}}^{2}}{m_{\mathrm{Rb}}+m_{\mathrm{Sr}}}}$. The third term is the kinetic energy of the relative motion. $\hat{H}_{\text {rot }}=\frac{\hbar^{2} \vec{\ell}^{2}}{2 \mu R^{2}}$ is the relative rotational Hamiltonian of the atomic pair with the reduced mass $\mu$, and $\vec{\ell}$ is the angular momentum. $\hat{H}_{\mathrm{el}}$ is the electronic Hamiltonian and $\hat{H}_{\mathrm{SO}}$ is the spin-orbit coupling. The term $\frac{1}{2} \mu \omega_{\mathrm{rel}}^{2} R^{2}$ is the harmonic potential determining the relative motion in the trap with the frequency $\omega_{\text {rel }}=\sqrt{\frac{m_{\mathrm{Sr}} \omega_{\mathrm{Rb}}^{2}+m_{\mathrm{Rb}} \omega_{\mathrm{Sr}}^{2}}{m_{\mathrm{Rb}}+m_{\mathrm{Sr}}}}$. 


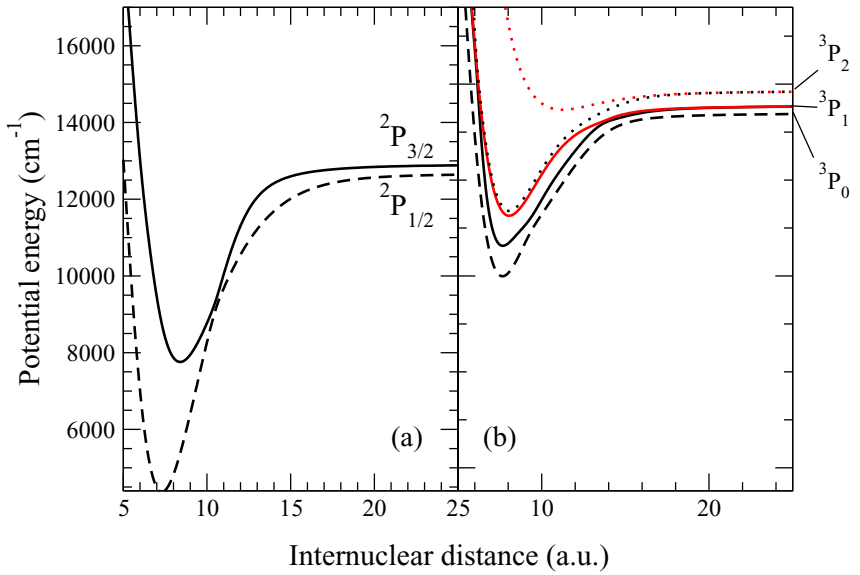

FIG. 2. Potential energy curves [59] for the excited electronic states of $\mathrm{RbSr}$, including spin-orbit couplings. (a) Electronic states correlated to the $\operatorname{Rb}\left(5 p^{2} P_{1 / 2,3 / 2}\right)+\operatorname{Sr}\left(5 s^{2}{ }^{1} S\right)$ asymptotes; (b) electronic states correlating to the $\mathrm{Rb}\left(5 s^{2} S\right)+\operatorname{Sr}\left(5 s 5 p^{3} P_{0,1,2}\right)$ asymptotes.

The last term is a dynamical term coupling the two motions, with $\Delta \omega=\sqrt{\omega_{\mathrm{Sr}}^{2}-\omega_{\mathrm{Rb}}^{2}}$.

Neglecting the latter coupling term turns out to be a crucial issue in order to avoid an otherwise complicated theoretical description of the atomic pair. Fortunately, Saenz et al. [56-58] give some regimes where the dynamical term is small so that the separation of the two motions remains a good approximation. This is precisely the case when the ratio $\frac{a}{a_{\omega}}$ is small, where $a_{\omega}=\frac{\hbar}{\sqrt{\mu \omega_{\mathrm{rel}}}}$ is the characteristic length of the relative motion in the trap, and $a$ is the scattering length. For $\omega_{\text {rel }}=2 \pi \times 400 \mathrm{kHz}$, used in our calculation, $a_{\omega}=484 a_{0}$ is larger than the ${ }^{87} \mathrm{Rb}_{-}{ }^{84} \mathrm{Sr}$ scattering length, $a=90.9 a_{0}$ [59]. The separation of the two motions can then be taken as a good approximation.

The trap frequency $\omega_{\text {rel }}=2 \pi \times 400 \mathrm{kHz}$ is larger than the one commonly used in experiments, around $2 \pi \times 100 \mathrm{kHz}$. A value as large as $2 \pi \times 400 \mathrm{kHz}$ would require an increase of the trapping laser intensity by a factor 16 . Here we chose $\omega_{\text {rel }}=2 \pi \times 400 \mathrm{kHz}$ in order to reduce the laser intensity for the presented transfer methods, and to avoid perturbation from other trap levels.

\section{B. Methodology for the solution of the relative motion equation}

After the separation of motions, the problem of the relative motion must be solved, with the Hamiltonian

$$
\hat{H}_{\text {trap }}^{\text {rel }}=-\frac{\hbar^{2}}{2 \mu} \nabla_{R}^{2}+\hat{H}_{\text {rot }}+\hat{H}_{\text {el }}+\hat{H}_{\text {SO }}+\frac{1}{2} \mu \omega_{\text {rel }}^{2} R^{2} .
$$

The electronic structure calculations have been described in detail in our previous papers [59,60]. A full configuration interaction (FCI) method involving the effective-core potential (ECP) and the core-polarization potential (CPP) was used. The inclusion of the spin-orbit coupling is also explained in these papers. The excited electronic states relevant for our study are shown in Fig. 2. They are described in the Hund case (c), and characterized by the absolute value of the projection of the total electronic angular momentum on the internuclear
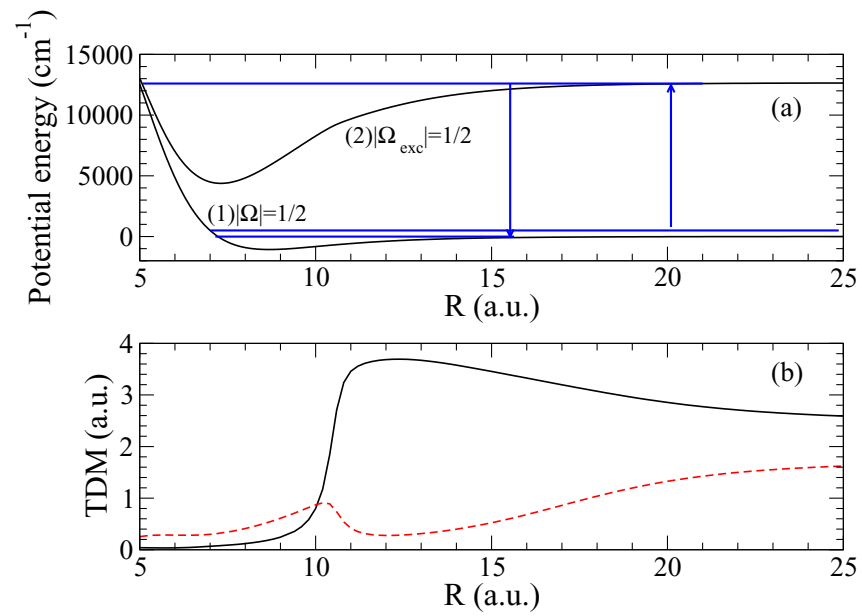

FIG. 3. (a) Potential energy curves [59] involved in the first STIRAP transfer between the lowest trap level and a high rovibrational level of the electronic ground state via a rovibrational level of an excited electronic state [(2) $\Omega=1 / 2]$. (b) Parallel component $\left\langle X^{2} \Sigma\left|\mu_{\|}\right|(2)^{2} \Sigma\right\rangle$ (in black) and perpendicular component $\left\langle X^{2} \Sigma\left|\mu_{\perp}\right|(1)^{2} \Pi\right\rangle$ (in dashed red) of the transition dipole moment (TDM) between the ground and excited electronic states.

axis, $|\Omega|$. They correlate to two groups of asymptotes: $\operatorname{Rb}(5 p$ $\left.{ }^{2} P_{1 / 2,3 / 2}\right)+\operatorname{Sr}\left(5 s^{2}{ }^{1} S\right)$ and $\operatorname{Rb}\left(5 s^{2} S\right)+\operatorname{Sr}\left(5 s 5 p{ }^{3} P_{0,1,2}\right)$. The Schrödinger equation for the nuclear relative motion is solved with the mapped Fourier grid Hamiltonian method (MFGH) [61-70], using a grid extending from $R_{\min }=5 a_{0}$ to $R_{\max }=$ $5000 a_{0}$, covering the spatial extension of the lowest trap level. This grid is the same for the ground and excited states in order to calculate the integral of the transition dipole moment between the bound rovibrational levels of electronic states.

\section{LASER CONTROL INVOLVING TWO ELECTRONIC STATES}

The first strategy studied in this paper for the formation of ultracold ${ }^{87} \mathrm{Rb}^{84} \mathrm{Sr}$ molecules is a variant of STIRAP relying on five levels. In our previous paper [60], we identified two possible STIRAP implementations, one for the chemical bond formation (see Fig. 3) and one for the vibrational quenching (see Fig. 4). After the first step, the molecules are created in a loosely bound level, as in magnetoassociation. The goal of the second step is to transfer the population of the loosely bound molecules toward the rovibrational ground level. The complete story line of the strategy is sketched in Fig. 1(a). When the two STIRAPs are applied consecutively, the process is called the two-consecutive STIRAP (tc-STIRAP)

During the STIRAP sequence, the molecules occupy a loosely bound level, and that could induce some problems. More precisely, the loosely bound molecules can be dissociated by photon scattering or molecule-molecule scattering. After dissociation, the atoms are usually lost from the trap. Molecule-molecule scattering is suppressed in the MI, the tunneling of ultracold particles between sites being negligible. To further limit losses, we have explored two methods that reduce the population in the loosely bound level during the STIRAP sequences: alternating-STIRAP (A-STIRAP) and straddle-STIRAP (S-STIRAP) [35-37]. In the present 

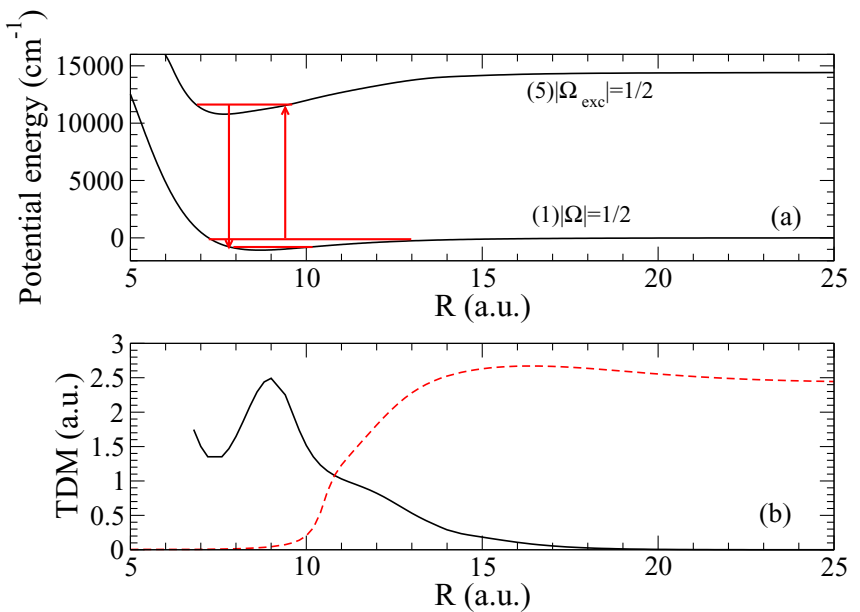

FIG. 4. (a) Potential energy curves [59] involved in the second STIRAP transfer between a high rovibrational level of the electronic ground state and the rovibrational ground level via a rovibrational level of an excited electronic state [(5) $\Omega=1 / 2]$. (b) Parallel component $\left\langle X^{2} \Sigma\left|\mu_{\|}\right|(3)^{2} \Sigma\right\rangle$ (in black), and perpendicular component $\left\langle X^{2} \Sigma\left|\mu_{\perp}\right|(2)^{2} \Pi\right\rangle$ (in dashed red) of the transition dipole moments between the electronic ground and the excited states.

case, they correspond to transfers between five levels via four pulses. An S-STIRAP strategy has already been used in the formation of ultracold $\mathrm{Cs}_{2}$ dimers [71]. The five levels are those of the tc-STIRAP: the lowest trap level $(N=0, J=$ $1 / 2)$ of the electronic ground state, the vibrational level $\left(v^{\prime}=\right.$ $199, J^{\prime}=3 / 2$ ) of the electronic state (2) $\left|\Omega_{\text {exc }}\right|=1 / 2$, the vibrational level $(v=-3, J=1 / 2)$ of the electronic ground state, the vibrational level $\left(v^{\prime}=15, J^{\prime}=3 / 2\right)$ of the state (5) $\left|\Omega_{\text {exc }}\right|=1 / 2$, and finally the rovibrational ground level $(v=$ $0, J=1 / 2$ ) (negative quantum numbers are counted from the dissociation limit). They are written, respectively, as follows: $\left|\Phi_{N=0, J=1 / 2}^{g}\right\rangle,\left|\Phi_{v^{\prime}=199, J^{\prime}=3 / 2}^{(2)\left|\Omega_{\text {exc }}\right|=1 / 2}\right\rangle,\left|\Phi_{v=-3, J=1 / 2}^{g}\right\rangle,\left|\Phi_{v^{\prime}=15, J^{\prime}=3 / 2}^{(5)\left|\Omega_{\text {exc }}\right|=1 / 2}\right\rangle$, and $\left|\Phi_{v=0, J=1 / 2}^{g}\right\rangle$. As illustrated in our paper [60], the first pump transition involves a loosely bound level of an excited electronic state correlated to the asymptote $\mathrm{Rb}\left(5 p^{2} P_{1 / 2}\right)+\mathrm{Sr}$ $\left(5 s^{2}{ }^{1} S\right)$ and not to the $\operatorname{Rb}\left(5 s^{2} S\right)+\operatorname{Sr}\left(5 s 5 p^{3} P_{0,1,2}\right)$ asymptotes (corresponding to the intercombination line of the strontium). At the large internuclear distance where this first pump transition occurs, the atomic character is dominant. On the other hand, the second pump transition involves a deeply bound level of an excited electronic state correlated to the $\mathrm{Rb}(5 \mathrm{~s}$ $\left.{ }^{2} S\right)+\operatorname{Sr}\left(5 s 5 p^{3} P_{1}\right)$ asymptote. The molecular field breaks the atomic spherical symmetry, and the atomic selection rule is no longer applied for deeply bound levels, inducing a large oscillator strength.

Like in the usual three-level STIRAP sequence, the methods are based on the existence of a dark state $|0\rangle$ in the field-dressed-state representation involving the five levels. Within the rotating wave approximation, it is given by

$$
\begin{aligned}
|0\rangle=\frac{1}{\mathcal{N}} & {\left[\Omega_{D_{1}}(t) \Omega_{D_{2}}(t)\left|\Phi_{N=0, J=1 / 2}^{g}\right\rangle\right.} \\
& -\Omega_{P_{1}}(t) \Omega_{D_{2}}(t)\left|\Phi_{v=-3, J=1 / 2}^{g}\right\rangle \\
& \left.+\Omega_{P_{1}}(t) \Omega_{P_{2}}(t)\left|\Phi_{v=0, J=1 / 2}^{g}\right\rangle\right]
\end{aligned}
$$

where $\Omega_{P_{i}\left(D_{i}\right)}$ are the Rabi frequencies for the pump $(P)$ and dump $(D)$ transitions of the $i$ th $\operatorname{STIRAP}(i=1,2) . \mathcal{N}$ is a normalization factor. They are calculated from the molecular transition dipole matrix elements (TDM). After some angular momentum algebra, we obtain the following expression for the TDM with linear polarization $\hat{\epsilon}_{0}$ :

$$
\begin{aligned}
\left|\left\langle\Phi_{v, J=1 / 2}^{g}\left|\vec{\mu} \cdot \hat{\epsilon}_{0}\right| \Phi_{v^{\prime}, J^{\prime}=3 / 2}^{(n)\left|\Omega_{\text {exc }}\right|=1 / 2}\right\rangle\right|^{2} \\
=\frac{2}{9}\left|\left\langle\phi_{v, J=1 / 2}^{X^{2} \Sigma}\left|\left\langle X^{2} \Sigma\left|\mu_{\|}\right|\left(n_{\Sigma}\right)^{2} \Sigma\right\rangle\right| \phi_{v^{\prime}, J=3 / 2}^{\left(n_{\Sigma}\right)^{2} \Sigma}\right\rangle\right|^{2} \\
\quad+\frac{1}{9}\left|\left\langle\phi_{v, J=1 / 2}^{X^{2} \Sigma}\left|\left\langle X^{2} \Sigma\left|\mu_{\perp}\right|\left(n_{\Pi}\right)^{2} \Pi\right\rangle\right| \phi_{v^{\prime}, J^{\prime}=3 / 2}^{\left(n_{\Pi}\right)^{2} \Pi}\right\rangle\right|^{2},
\end{aligned}
$$

where $\mu_{\|}$and $\mu_{\perp}$ are the parallel and perpendicular components of the electric dipole moment. The levels are expressed in the Hund case (a) basis: $\left|\Phi_{v, J=1 / 2}^{g}\right\rangle=$ $\left|\phi_{v, J=1 / 2}^{X^{2} \Sigma}\right\rangle\left|X^{2} \Sigma\right\rangle$ for the electronic ground state and $\left|\Phi_{v^{\prime}, J_{\mathrm{exc}}=3 / 2}^{(n)\left|\Omega_{\mathrm{exc}}\right|=1 / 2}\right\rangle=\left|\phi_{v^{\prime}, J^{\prime}=3 / 2}^{\left(n_{\Sigma}\right)^{2} \Sigma}\right\rangle\left|\left(n_{\Sigma}\right)^{2} \Sigma\right\rangle+\left|\phi_{v^{\prime}, J^{\prime}=3 / 2}^{\left(n_{\Pi}\right)^{2} \Pi}\right\rangle\left|\left(n_{\Pi}\right)^{2} \Pi\right\rangle$ for the electronic excited states $(n=2$ or 5$)$. The quantity $\left|\phi_{v^{\prime}, J^{\prime}=3 / 2}^{\left(n_{\Sigma}\right)^{2} \Sigma}\right\rangle$ is the component of this level on the corresponding ${ }^{2} \Sigma$ state, with a similar notation for the ${ }^{2} \Pi$ state. For the (2) $\left|\Omega_{\text {exc }}\right|=1 / 2$ electronic state, $n_{\Sigma}=2$ and $n_{\Pi}=1$ while for the (5) $\left|\Omega_{\text {exc }}\right|=1 / 2$ electronic state, $n_{\Sigma}=3$ and $n_{\Pi}=2$. The former induces a coupling with the ${ }^{2} \Sigma$ component of the electronic excited states, while the latter leads to a coupling with their ${ }^{2} \Pi$ component. The matrix elements $\left\langle X^{2} \Sigma\left|\mu_{\|}\right|\left(n_{\Sigma}\right)^{2} \Sigma\right\rangle$ and $\left\langle X^{2} \Sigma\left|\mu_{\perp}\right|\left(n_{\Pi}\right)^{2} \Pi\right\rangle$ for the involved excited electronic states are represented in Figs. 3(b) and 4(b). Note that in addition to what has been done in our previous paper [60], here we include the rotational contributions. The values (in a.u.) of the TDM for the four transitions are the following: $\left|\mu_{P_{1}}\right|^{2}=$ $5.2 \times 10^{-5},\left|\mu_{D_{1}}\right|^{2}=1.3 \times 10^{-4},\left|\mu_{P_{2}}\right|^{2}=5.5 \times 10^{-5}$, and $\left|\mu_{D_{2}}\right|^{2}=9.8 \times 10^{-5}$.

The A-STIRAP consists in applying the two dump pulses (second and fourth transitions) prior to the two pump pulses (first and third transitions). We have simulated the dynamics of the A-STIRAP process. In the interaction representation, the total wave function is expressed on a basis composed of rovibrational and trap levels of the electronic states $\left|\Phi_{v_{k}, J_{k}}^{\mathrm{el}_{k}}\right\rangle$ as

$$
|\Psi(t)\rangle=\sum_{k} \tilde{C}_{k}(t) e^{-\frac{i}{\hbar} E_{k} t}\left|\Phi_{v_{k}, J_{k}}^{\mathrm{el}_{k}}\right\rangle .
$$

The time-dependent coefficients $\tilde{C}_{k}(t)$ are obtained by resolution of the coupled equations

$$
i \hbar \frac{d \tilde{C}_{k}}{d t}=-\sum_{j} \mu_{k j} \mathcal{E}(t) e^{\frac{i}{\hbar}\left(E_{k}-E_{j}\right) t} \tilde{C}_{j}(t),
$$

where $\mu_{k j}=\left\langle\Phi_{v_{k}, J_{k}}^{\mathrm{el}_{k}}\left|\vec{\mu} \cdot \hat{\epsilon}_{0}\right| \Phi_{v_{j}, J_{j}}^{\mathrm{el}_{j}}\right\rangle$.

The propagation of these coupled equations is made with a Runge-Kutta 4 (RK4) algorithm with a time step of 24 fs. The field $\mathcal{E}(t)$ comprises four pulses,

$$
\begin{aligned}
\mathcal{E}(t)= & \mathcal{E}_{P_{1}} \exp \left(-\frac{\left(t-t_{c}^{P_{1}}\right)^{2}}{\tau_{P_{1}}^{2}}\right) \cos \left(\omega_{P_{1}} t\right) \\
& +\mathcal{E}_{D_{1}} \exp \left(-\frac{\left(t-t_{c}^{D_{1}}\right)^{2}}{\tau_{D_{1}}^{2}}\right) \cos \left(\omega_{D_{1}} t\right)
\end{aligned}
$$



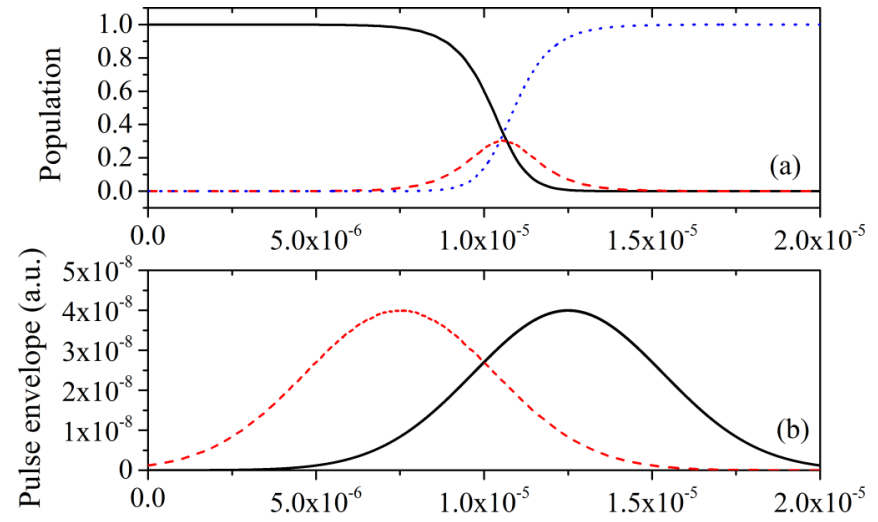

$\mathrm{t}(\mathrm{s})$

FIG. 5. (a) Simulation of population transfer from the lowest trap level (black) to the rovibrational ground level (dotted blue) via the loosely bound rovibrational level $v=-3$ (dashed red). (b) Envelope of the pump (black) and dump (dashed red) fields of the A-STIRAP process.

$$
\begin{aligned}
& +\mathcal{E}_{P_{2}} \exp \left(-\frac{\left(t-t_{c}^{P_{2}}\right)^{2}}{\tau_{P_{2}}^{2}}\right) \cos \left(\omega_{P_{2}} t\right) \\
& +\mathcal{E}_{D_{2}} \exp \left(-\frac{\left(t-t_{c}^{D_{2}}\right)^{2}}{\tau_{D_{2}}^{2}}\right) \cos \left(\omega_{D_{2}} t\right)
\end{aligned}
$$

where $\mathcal{E}_{\left(P_{i}, D_{i}\right)}$ are the maximum field amplitudes, $t_{c}^{\left(P_{i}, D_{i}\right)}$ are the Gaussian pulse central times, $\tau_{\left(P_{i}, D_{i}\right)}$ are the temporal widths, and $\omega_{\left(P_{i}, D_{i}\right)}$ are the frequencies of the transitions $(i=1,2)$. The simulation of the population dynamics is conducted with a basis of 30 levels made of the five levels of the A-STIRAP transfer, to which are added 19 excited trap levels $(N=$ $1-19, J=1 / 2)$, the rovibrational levels $\left(v^{\prime}=198, J=\right.$ $3 / 2)$ and $\left(v^{\prime}=200, J=3 / 2\right)$ of (2) $\left|\Omega_{\text {exc }}\right|=1 / 2$, the vibrational levels $\left(v^{\prime}=14, J=3 / 2\right)$ and $\left(v^{\prime}=16, J=3 / 2\right)$ of (5) $\left|\Omega_{\text {exc }}\right|=1 / 2$, and the vibrational levels $(v=-4, J=$ $1 / 2)$ and $(v=-2, J=1 / 2)$ of the electronic ground state. A complete transfer is achieved for a total duration of the pulse sequence of $20 \mu \mathrm{s}$ (Fig. 5). The amplitudes of the pulses are $4.0 \times 10^{-8}$ a.u. (or intensities of $306 \mathrm{~W} / \mathrm{cm}^{2}$ ). Such intensities are potentially reachable in current ultracold experiments. The other field parameters are $t_{c}^{P_{1}}=t_{c}^{P_{2}}=12.5 \mu \mathrm{s}$, $t_{c}^{D_{1}}=t_{c}^{D_{2}}=7.5 \mu \mathrm{s}$, and $\tau_{P_{1}}=\tau_{P_{2}}=\tau_{D_{1}}=\tau_{D_{2}}=4.0 \mu \mathrm{s}$.

Unfortunately, the A-STIRAP does not solve completely the issue of the occupation of the loosely bound level $(v=-3)$. This is expected since this level has a component in the dark state 3 . The maximum population of this level during the dynamics is $30 \%$, which is still an improvement with respect to the tc-STIRAP, where this level is $100 \%$ populated between the two STIRAPs.

With the S-STIRAP, we can go further since we succeeded in minimizing the transient population in the loosely bound level. In this case, the second (first dump) and third (second pump) pulses have higher amplitudes and completely overlap the first (first pump) and the fourth (second dump) pulses [Fig. 6(b)]. Again, we obtain an efficient transfer for a pulse sequence of $20 \mu \mathrm{s}$. The parameters of the pulses are $\mathcal{E}_{P_{1}}=$ $5.0 \times 10^{-8}$ a.u., $t_{c}^{P_{1}}=12.5 \mu \mathrm{s}, \tau_{P_{1}}=5.7 \mu \mathrm{s} ; \mathcal{E}_{D_{1}}=8.0 \times$
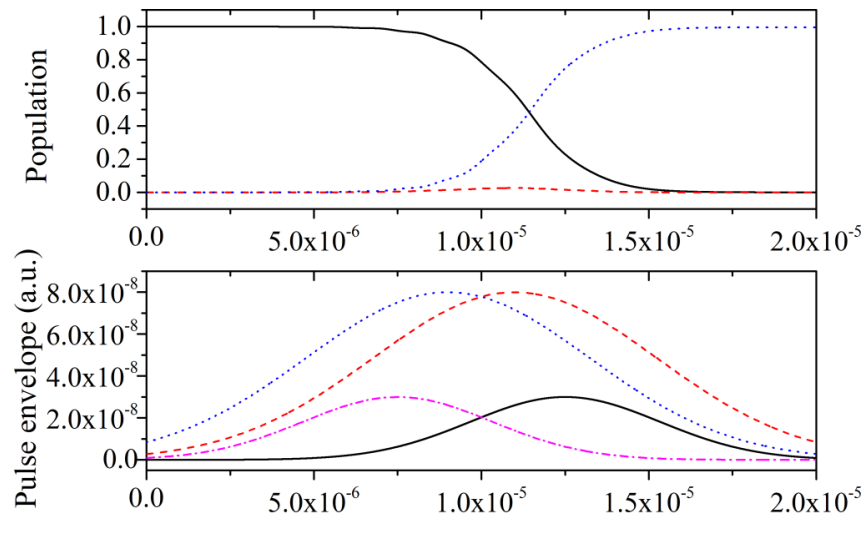

$\mathrm{t}(\mathrm{s})$

FIG. 6. (a) Simulation of population transfer from the lowest trap level (black) to the rovibrational ground level (dotted blue) via the loosely bound rovibrational level $v=-3$ (dashed red). (b) Envelope of the first pump (black), first dump (dotted blue), second pump (dashed red), and second dump (dot-dashed magenta) fields of the S-STIRAP process.

$10^{-8}$ a.u., $t_{c}^{D_{1}}=9 \mu \mathrm{s}, \tau_{D_{1}}=8.5 \mu \mathrm{s} ; \mathcal{E}_{P_{2}}=8.0 \times 10^{-8}$ a.u., $t_{c}^{P_{2}}=11 \mu \mathrm{s}, \tau_{P_{2}}=8.5 \mu \mathrm{s}$; and $\mathcal{E}_{D_{2}}=5.0 \times 10^{-8}$ a.u., $t_{c}^{D_{2}}=$ $7.5 \mu \mathrm{s}, \tau_{D_{2}}=5.7 \mu \mathrm{s}$. The peak intensities are $1220 \mathrm{~W} / \mathrm{cm}^{2}$, which are more challenging to achieve for such a pulse duration. However, the S-STIRAP allows minimizing the population in the loosely bound level $(v=-3)$. The maximum population in this level is $2.7 \%$ and could be further decreased by increasing the amplitudes $\mathcal{E}_{D_{1}}$ and $\mathcal{E}_{P_{2}}$. In conclusion, the S-STIRAP seems to be the best solution if the lifetime of the loosely bound level is very short and is a strong constraint on the transfer. The cost would be the use of strong field intensities. In other situations, the A-STIRAP seems to be the most appropriate method since it requires lower laser intensities. Note that for a trap frequency $\omega_{\text {rel }}=2 \pi \times 100 \mathrm{kHz}$, the first transition is perturbed by the presence of other trap states, and for solving this issue the best solution is increasing the duration of the transfer to $200 \mu \mathrm{s}$.

\section{LASER CONTROL INVOLVING A SINGLE ELECTRONIC (GROUND) STATE}

Another solution for avoiding the spontaneous emission is to restrict the dynamics within the electronic ground state. The formation of heteronuclear molecules is possible via this strategy thanks to the presence of a permanent dipole moment in the electronic ground state of RbSr [Fig. 7(b)]. Unfortunately, the matrix element between the lowest trap level and the rovibrational ground level is too small $\left(10^{-20}\right.$ a.u. $)$ to induce a direct transfer. As for the STIRAP, the strategy must be divided into two steps: the molecular bond formation and the vibrational quenching. Approaches presenting some similarities and relying on a single electronic state photoassociation have also been worked out for ultracold LiCs formation [72].

\section{A. Molecular bond formation}

First, we must identify the final rovibrational level. The criterion for that purpose is simply the vibrational level with 

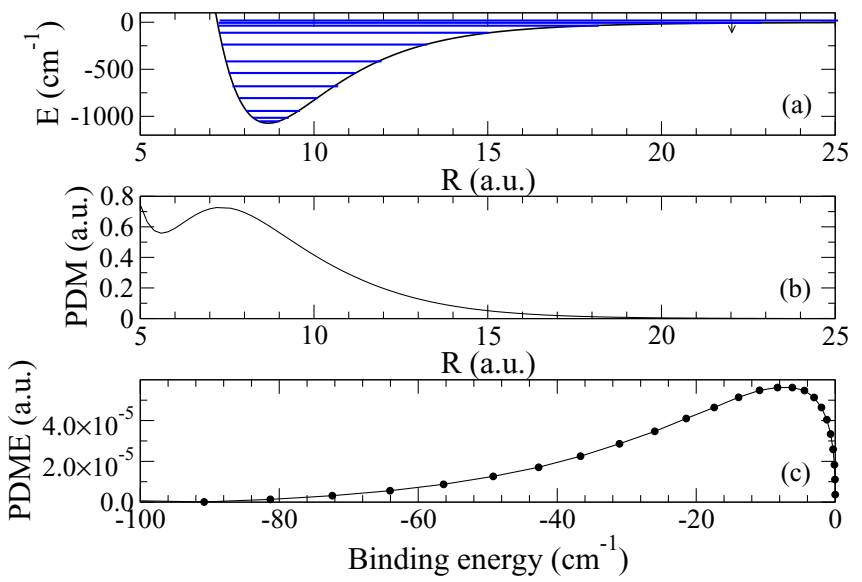

FIG. 7. (a) Potential energy curve [59] of the electronic ground state of RbSr. Vibrational levels that are involved in the transfer to the rovibrational ground level are drawn with blue solid lines. (b) Permanent dipole moment (PDM) $\left\langle X^{2} \Sigma\left|\mu_{\|}\right| X^{2} \Sigma\right\rangle$ of the $\mathrm{RbSr}$ electronic ground state. (c) PDM matrix element (PDMME) between the lowest trap level $N=0$ and the vibrational levels of the electronic ground state (without rotation).

the highest PDM matrix element with the lowest trap level, which is the vibrational level $v=56$ [73] [Fig. 7(c)]. Concerning the rotation, the largest angular factors are obtained for a transition $J=1 / 2 \rightarrow 3 / 2$. For a linear polarization, the angular factor is $\sqrt{2} / 3$. Two types of pulses are considered for the transfer: a chirped pulse and a $\pi$-pulse.

\section{Chirped pulse}

The principle of the transfer is the adiabatic passage between the two levels via a chirped pulse $[74,75]$. In the dressed-state representation, a frequency tuning across the resonance induces a crossing of the two levels, but an avoided crossing between the two adiabatic levels. When the frequency is slowly swept such that the system always stays in the same adiabatic level, the population is transferred from the lowest trap level into the target rovibrational level.

We note that this method of formation follows the same principle as magnetoassociation based on a magnetic Feshbach resonance. In a similar way, the laser coupling between the trapping level and the vibrational level can be described as an optical Feshbach resonance, which we recently identified as a laser-assisted self-induced Feshbach resonance (LASIFR) [73]. In addition to the molecule formation, the scattering length can be controlled by changing the frequency. Conceptually, the LASIFR concept leads to the idea that the field-coupling of continuum levels with a bound level of the same electronic state can be related to a Feshbach resonance in the dressed-molecular-state representation.

The formation of the molecule is conveniently described by the Landau-Zener (LZ) model:

$$
\begin{aligned}
p_{\text {trap }} & =\exp \left(-\pi \frac{\Omega_{\mathrm{LASIFR,max}}^{2}}{2|\alpha|}\right), \\
p_{v} & =1-\exp \left(-\pi \frac{\Omega_{\mathrm{LASIFR}, \max }^{2}}{2|\alpha|}\right),
\end{aligned}
$$

TABLE I. Values of the parameters inducing a population of $99 \%$ in the rovibrational level $(v=56, J=3 / 2)$ via an adiabatic passage from the lowest trap level $(N=0, J=1 / 2)$. $\alpha$ is the chirp rate, $I_{0} \propto$ $\mathcal{E}_{0}^{2}$ is the peak intensity, and $t_{\max }$ is the duration of the pulse. The laser is linearly polarized.

\begin{tabular}{lcr}
\hline \hline$t_{\max }(\mu \mathrm{s})$ & $|\alpha|(\mathrm{MHz} / \mu \mathrm{s})$ & $I_{0}\left(\mathrm{~W} / \mathrm{cm}^{2}\right)$ \\
\hline 100 & $1.0 \times 10^{-2}$ & 30000 \\
100 & $5.0 \times 10^{-3}$ & 15000 \\
10000 & $1.0 \times 10^{-4}$ & 300 \\
10000 & $5.0 \times 10^{-5}$ & 150 \\
10000 & $5.0 \times 10^{-5}$ & 225 \\
\hline \hline
\end{tabular}

where $p_{v}$ is the final probability of the vibrational level, $p_{\text {trap }}$ is the final probability of the trap level, and $\Omega_{\text {LASIFR,max }}$ is the maximum Rabi frequency at the avoided crossing. The linear chirp rate $\alpha$ is defined by

$$
\alpha=\frac{\omega_{f}-\omega_{0}}{t_{\max }},
$$

where $\omega_{0, f}$ are the initial and final frequency, respectively, and $t_{\max }$ is the duration of the pulse. However, the LZ model presents limitations related to the two-state assumption: only the initially populated trap level and the target vibrational level are considered. Actually, the transfer can be perturbed by other levels, and especially by the other trap levels. A first consequence of the inclusion of trap levels is the asymmetry in the sign of the chirp rate $\alpha$. An efficient transfer can only be obtained for a negative ramp. A positive ramp induces the transfer to the other trap level and hence induces a heating of the ultracold gas. For a negative ramp, there is also a limitation due to the last vibrational level. If the variation of the frequency is greater than the binding energy of the last vibrational level, the population is transferred to this level and not to the target vibrational level. These constraints on the sign and the magnitude of the ramp are also observed for magnetoassociation [26]. Furthermore, the presence of the other trap level also induces a Stark shift on the two levels involved in the adiabatic passage. The value of this Stark shift can be reduced with a high trap frequency, as is the MI case. The increase of the trap frequency also allows the increase of the coupling between the lowest trap level and the target vibrational level $(v=56)$.

The parameter values of the LZ model (8) are reported in Table I for a linear polarization. The adiabaticity criterion is fulfilled when $\Omega_{\text {LASIFR,max }}^{2} \gg \alpha$. The comparison between transfer strategies is made referring to the maximal intensity, which is the main experimental constraint. For an intensity similar to that used for the STIRAP (hundreds of $\mathrm{W} / \mathrm{cm}^{2}$ ), a pulse duration of $10 \mathrm{~ms}$ is necessary. The long duration of the pulse is due to the small values of the PDM matrix elements compared to those of the transition dipole moments. An interesting advantage with respect to the tc-STIRAP (Sec. IV) is that the created molecules occupy a more bound rovibrational level ( $v=56$ versus $v=63$ ), less sensitive to losses. Finally, we note that a decrease of the trap frequency to $\omega_{\text {rel }}=2 \pi \times 100 \mathrm{kHz}$ would induce an increase of the $\mathrm{THz}$ intensity $I_{0}$ by a factor 4 . 
TABLE II. Values of the parameters of a Gaussian pulse for obtaining a $\pi$-pulse inducing the transition from the trap level $(N=0, J=1 / 2)$ to the rovibrational level $(v=56, J=3 / 2)$. A linear polarization is used.

\begin{tabular}{lrcr}
\hline \hline$t_{c}(\mu \mathrm{s})$ & $\tau(\mu \mathrm{s})$ & $\mathcal{E}_{0}$ (a.u.) & $I_{0}\left(\mathrm{~W} / \mathrm{cm}^{2}\right)$ \\
\hline 5 & 2.0 & $1.09 \times 10^{-6}$ & 123987 \\
10 & 3.4 & $5.44 \times 10^{-7}$ & 30997 \\
25 & 9.9 & $2.18 \times 10^{-7}$ & 4959 \\
50 & 19.8 & $1.09 \times 10^{-7}$ & 1240 \\
100 & 39.6 & $5.44 \times 10^{-8}$ & 310 \\
250 & 99.0 & $2.18 \times 10^{-8}$ & 50 \\
500 & 198.0 & $1.09 \times 10^{-8}$ & 12 \\
5000 & 1980.0 & $1.09 \times 10^{-9}$ & 0.12 \\
\hline
\end{tabular}

\section{2. $\pi$-pulse}

An alternative to a chirped pulse is a $\pi$-pulse [76,77], such as the temporal integral of the Rabi frequency, which is a multiple of $\pi$. In contrast with the chirped pulse, the frequency is fixed and kept at resonance. The field has a Gaussian shape like in Eq. (7), with similar parameter definitions,

$$
\mathcal{E}(t)=\mathcal{E}_{0} \exp \left(-\frac{\left(t-t_{c}\right)^{2}}{\tau^{2}}\right) \cos \left(\omega_{0} t\right) .
$$

For such a linearly polarized pulse, we can derive an analytical formula for the conditions of a complete transfer,

$$
\mathcal{E}_{0} \cdot \tau=\frac{\sqrt{\pi}}{\left\langle\phi_{v_{\text {res }}=56, J=3 / 2}^{g}\left|\left\langle X^{2} \Sigma\left|\mu_{\|}\right| X^{2} \Sigma\right\rangle\right| \phi_{N=0, J=1 / 2}^{g}\right\rangle} .
$$

In Table II, we display the parameters $\left(\mathcal{E}_{0}, \tau\right)$ that fulfill the condition of Eq. (11). The required intensities are lower than those for the chirped pulses. As the intensity is a major limitation for THz sources, it is a clear advantage for $\pi$-pulses with respect to the chirped ones. On the other hand, the $\pi$-pulses are more sensitive to noise sources affecting laser parameters. The adiabatic passage is a more robust method. In conclusion, the $\pi$-pulse appears as the more feasible and the more realistic method, but we can anticipate that with the development of $\mathrm{THz}$ sources, chirped pulses could become the preferred method.

Finally, we have simulated the $\pi$-pulse dynamics with the parameters of Table II. We follow the same methodology as for the simulation of the STIRAP process. We used an extended basis of 20 trap levels $(N=0-19, J=1 / 2)$ and 4 vibrational levels $(v=55,56,57$, and $65, J=3 / 2)$. The results for $\tau=19.8 \mu \mathrm{s}$ and $\mathcal{E}_{0}=1.09 \times 10^{-7}$ a.u. are reported in Fig. 8. The final population in the target vibrational level is $98.8 \%$, illustrating the efficiency of the transfer. The perturbation by the other trap levels is limited and does not affect the efficiency of the transfer. However, this statement is not true for the trap frequency $\omega_{\text {rel }}=2 \pi \times 100 \mathrm{kHz}$, and the other trap levels could affect the transfer.

\section{B. Vibrational quenching}

In this section, we examine the pros and cons of different strategies in the infrared domain that would in principle allow the transition toward the absolute ground rovibrational level

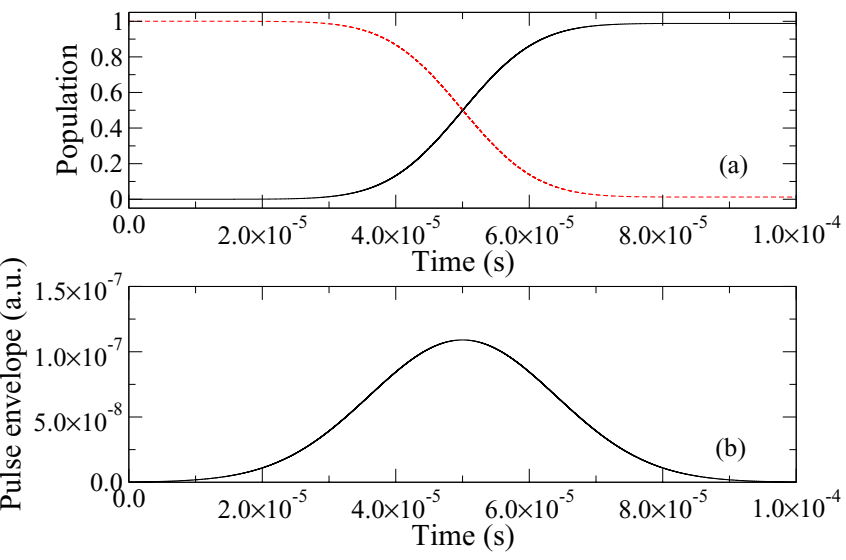

FIG. 8. (a) Evolution of the populations in the lowest trap level (black) and in the target rovibrational level (dashed red) induced by a $\pi$-pulse with the envelope shown in (b).

starting from the molecular bound level $v=56$ prepared in the previous subsection. However, generating shaped pulses in this spectral region remains very challenging nowadays. This simulation aims at analyzing the limits of some alternatives accounting for the complexity of the vibrational chain, of the wide order of magnitude of the dipolar coupling, and by using a reasonable intensity range. In a first attempt, we disregard the Clebsh-Gordan coefficients and the rotational degree of freedom. The basis set contains 80 levels, i.e., we retain the dense manifold above the $v=56$ level. The computation is carried out by the Runge-Kutta algorithm in interaction representation without the rotating wave approximation. The time step is calibrated from the highest frequency involved in this simulation and fixed to $0.12 \mathrm{ps}$. Figure 9 displays the matrix elements $\mu_{v v^{\prime}}$ of the permanent dipole moment.

We compare two strategies. In the first one, in order to minimize the number of pulses we select a chain of about ten levels for which the dipolar coupling is smaller than the value among neighboring levels, but is larger than $10^{-4}$ a.u. We then compare a series of $\pi$-pulses with an S-STIRAP sequence improved by optimal control theory (OCT) [78] according to the Rabitz iterative scheme [79], where the S-STIRAP field is taken as the guess field. In the second approach, we exploit all the successive neighboring levels by using a chirped pulse. The latter is also used as a guess field for improving the yield by OCT. Note that this step does not depend on the trap frequency. Indeed, the involved vibrational levels are sufficiently bound to be unaffected by a change of the trap frequency.

\section{Train of IR $\pi$-pulses}

The chosen intermediary transitions and the corresponding dipolar couplings are given in Table III of the Appendix. The electric fields are once more written as

$$
\mathcal{E}_{i j}(t)=\mathcal{E}_{0, i j} \exp \left[-\left(t-t_{c, i j}\right)^{2} / \tau_{i j}^{2}\right] \cos \left(\omega_{i j} t\right),
$$

where the indices $i j$ correspond to the transition $i \rightarrow j$.

In a first attempt, the leading amplitude is fixed at $10^{-5}$ a.u. (corresponding to $3.51 \times 10^{6} \mathrm{~W} / \mathrm{cm}^{2}$ ) for each transition, and the width $\tau_{i j}=\sqrt{\pi} /\left(\mu_{i j} \mathcal{E}_{0, i j}\right)$ is adjusted to satisfy the 

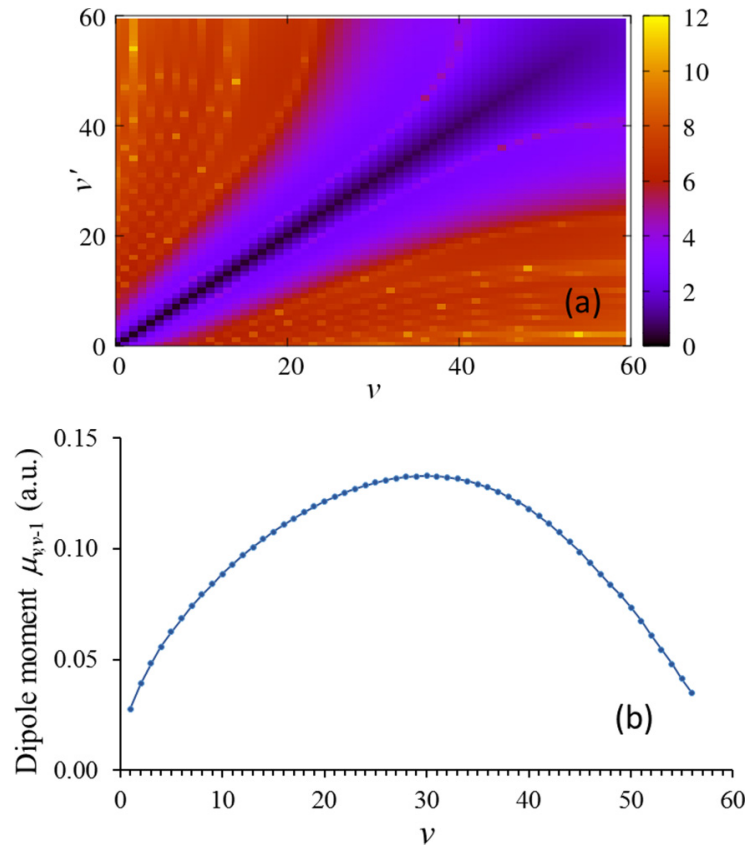

FIG. 9. (a) $-\log _{10}\left(\mu_{v v^{\prime}}\right)$ of the matrix elements of the dipole moment in a.u. for 60 levels for a trap of $400 \mathrm{kHz}$; (b) dipole moment $\mu_{v, v-1}$ between neighboring levels.

$\pi$-pulse condition in a.u.,

$$
\int_{t_{c, i j}-t_{\max , i j}}^{t_{c, i j}+t_{\max , i j}} \mu_{i j} \mathcal{E}_{i j}(t) d t=\pi .
$$

The final time $t_{\max , i j}$ for the simulations is taken as $t_{\max , i j}=$ $4 \sqrt{\ln (2)} \tau_{i j}$. As the couplings are varying by two orders of magnitude along the selected chain, the pulse durations strongly differ, and the width $\tau_{i j}$ is given in Table III of the Appendix. Obviously, an alternative would be to fix the pulse duration and let the amplitude vary. Each $\pi$-pulse ensures the selected transition without any perturbation due to the other levels of the basis set. We have concatenated all the pulses in order to check the stability and loss of phase relation since every simulation was independent, without control of the final phase of the probability amplitude in the final level.

TABLE III. Parameters of the transitions and widths of the $\pi$-pulses.

\begin{tabular}{lccc}
\hline \hline Transition & $\omega_{i j}\left(\mathrm{~cm}^{-1}\right)$ & Dipole $\mu_{i j}$ (a.u.) & $\tau_{i j}(\mathrm{~ns})$ \\
\hline $56-48$ & 30.5 & $1.446 \times 10^{-2}$ & 0.295 \\
$48-39$ & 75.1 & $2.267 \times 10^{-3}$ & 1.884 \\
$39-30$ & 125.9 & $1.563 \times 10^{-3}$ & 2.732 \\
$30-21$ & 178.7 & $7.305 \times 10^{-4}$ & 5.847 \\
$21-16$ & 123.7 & $1.807 \times 10^{-3}$ & 2.364 \\
$16-11$ & 141.0 & $1.196 \times 10^{-3}$ & 3.573 \\
$11-7$ & 125.5 & $1.096 \times 10^{-3}$ & 3.897 \\
$7-3$ & 136.9 & $4.485 \times 10^{-4}$ & 9.525 \\
$3-1$ & 72.6 & $1.749 \times 10^{-3}$ & 2.442 \\
$1-0$ & 37.3 & $2.767 \times 10^{-2}$ & 0.154 \\
$2-1$ & 36.6 & $3.925 \times 10^{-2}$ & \\
$3-2$ & 35.9 & $4.818 \times 10^{-2}$ & \\
\hline \hline
\end{tabular}
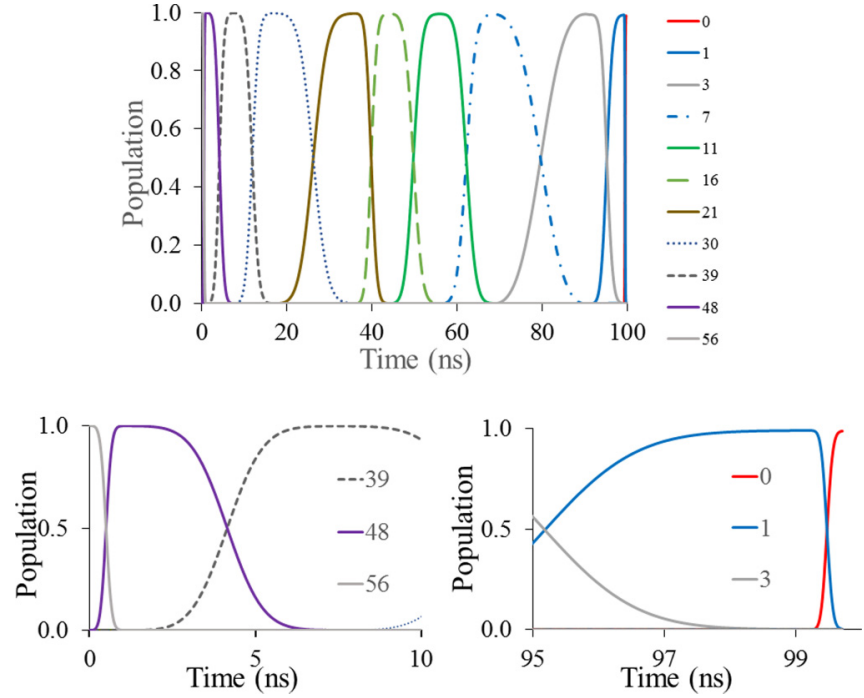

FIG. 10. Upper panel: Population evolution driven by concatenated $\pi$-pulses described in Table III of the Appendix. Lower panel: zoom on the two short pulses inducing the first and last transitions.

The vibrational ground level is reached within $98.9 \%$ when the full transition is driven by the concatenated field. The population evolution is displayed in Fig. 10. The complete transition requires about $100 \mathrm{~ns}$ with the chosen maximum amplitude. The first and last transitions have a short duration due to their high dipole moment.

\section{S-STIRAP and OCT}

We now explore another strategy inspired from the SSTIRAP scheme by involving a chain of intermediate levels as in the $\pi$-pulse sequence. Only two transitions $1-2$ and 2 -3 are added to select 11 active levels. The corresponding frequencies and dipole moments are given in Table III of the Appendix. The pulses inducing the extreme transitions $56-48$ and $1-0$ are driven in counterintuitive order and they are straddled by the nine pulses connecting the intermediate levels. The first set of parameters is given in Table IV of the Appendix. We fix the maximum field amplitude as in the $\pi$-pulse sequence, and we increase the total duration of the simulation $t_{\max }$ by keeping the relative gap between

TABLE IV. First parameter set of the S-STIRAP sequence.

\begin{tabular}{llll}
\hline \hline Transition & $t_{c} / t_{\max }$ & $\tau / t_{\max }$ & $\mathcal{E}_{0}$ (a.u.) \\
\hline $56-48$ & 0.58 & 0.2 & $10^{-6}$ \\
$48-39$ & 0.5 & 0.42 & $10^{-6}$ \\
$39-30$ & 0.5 & 0.42 & $10^{-6}$ \\
$30-21$ & 0.5 & 0.42 & $10^{-5}$ \\
$21-16$ & 0.5 & 0.42 & $10^{-5}$ \\
$16-11$ & 0.5 & 0.42 & $10^{-5}$ \\
$11-7$ & 0.5 & 0.42 & $10^{-5}$ \\
$7-3$ & 0.5 & 0.42 & $10^{-5}$ \\
$3-2$ & 0.5 & 0.42 & $10^{-6}$ \\
$2-1$ & 0.5 & 0.42 & $10^{-6}$ \\
$1-0$ & 0.42 & 0.2 & $10^{-6}$ \\
\hline
\end{tabular}



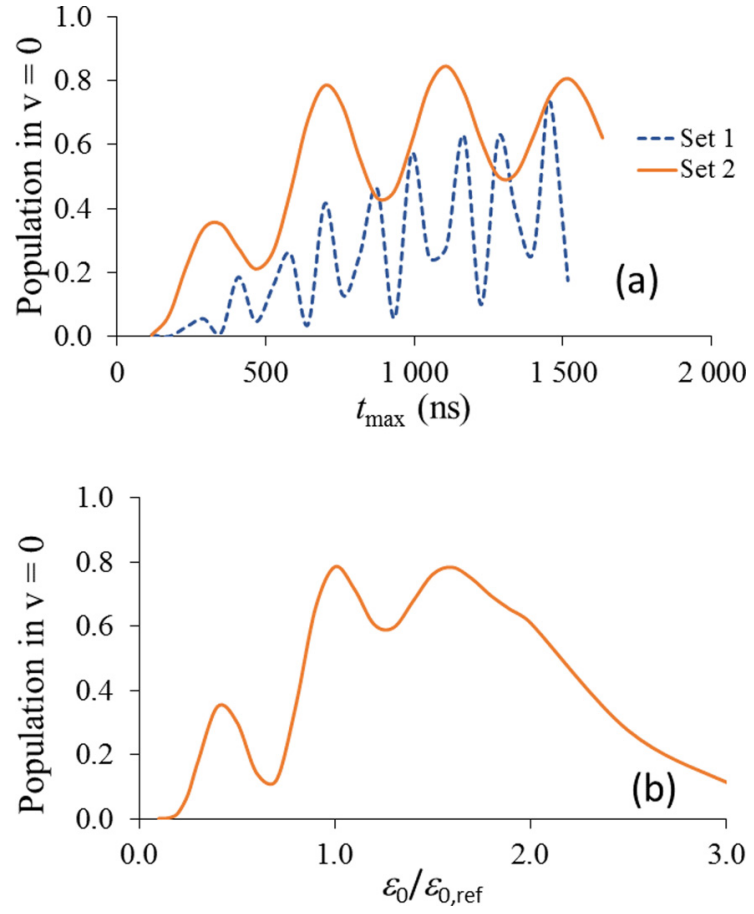

FIG. 11. (a) Population in the $v=0$ ground level as a function of the total sequence duration $t_{\max }$ for two parameter sets. Dashed line: set 1 given in Table IV of the Appendix. Full line: set 2 given in Table $\mathrm{V}$ of the Appendix. (b) Variation of the maximal amplitude of each pulse by the same factor $\mathcal{E}_{0} / \mathcal{E}_{0 \text {,ref }}$ for $t_{\text {max }}=700 \mathrm{~ns}$, where $\mathcal{E}_{0 \text {,ref }}$ designates the initial choice of the amplitudes given in Table $\mathrm{V}$ of the Appendix.

the pulse positions $t_{c, i j}$ and the relative widths $\tau_{i j}$. As the transfer occurs in the ground electronic level, the aim is not to completely avoid population in the intermediate levels since the radiative lifetimes are very long. We focus on the final yield in the ground $v=0$ level. The final population in the ground vibrational level as a function of $t_{\max }$ is drawn as a dashed line in Fig. 11(a). It increases on average, but strongly fluctuates and exceeds $50 \%$ for a long $t_{\max }$ of the order of $1 \mu \mathrm{s}$. The yield could increase with a longer duration, but it seems difficult to reach $100 \%$.

To reduce the total pulse duration, we choose a shorter $t_{\max }=700 \mathrm{~ns}$ corresponding to a local yield maximum of $40 \%$, and we slightly modify the pulse parameters, $t_{c}, \tau$, and $\mathcal{E}_{0}$. The parameters leading to $80 \%$ are given in Table $\mathrm{V}$ of the Appendix. The evolution of the yield with $t_{\max }$ by using this parameter set is shown by a full line in Fig. 11(a). We also analyze the influence of the maximum amplitude on the yield. Figure 11(b) shows the effect of varying all the amplitudes by a common factor. The population in $v=0$ is drawn as a function of $\mathcal{E}_{0} / \mathcal{E}_{0 \text {,ref }}$, where $\mathcal{E}_{0 \text {,ref }}$ is given in Table $\mathrm{V}$ of the Appendix.

Finally, starting again from the new reference parameter set giving a yield $80 \%$ for $t_{\max }=700 \mathrm{~ns}$ (Table $\mathrm{V}$ in the Appendix), we optimize the field by OCT. The field is discretized on a grid time $t_{i}$, and the process is initiated with a guess field, which is here S-STIRAP field. Note that the optimized parameters are all the values $\mathcal{E}\left(t_{i}\right)$, and not the parameters of the analytical expressions of the guess pulses. The
TABLE V. Second parameter set of the S-STIRAP sequence.

\begin{tabular}{lccr}
\hline \hline Transition & $t_{c} / t_{\max }$ & $\tau / t_{\max }$ & \multicolumn{1}{c}{$\mathcal{E}_{0}$ (a.u.) } \\
\hline $56-48$ & 0.58 & 0.2 & $3 \times 10^{-7}$ \\
$48-39$ & 0.38 & 0.42 & $10^{-6}$ \\
$39-30$ & 0.38 & 0.42 & $10^{-6}$ \\
$30-21$ & 0.5 & 0.42 & $1.16 \times 10^{-5}$ \\
$21-16$ & 0.5 & 0.42 & $1.16 \times 10^{-5}$ \\
$16-11$ & 0.5 & 0.42 & $1.16 \times 10^{-5}$ \\
$11-7$ & 0.5 & 0.42 & $1.16 \times 10^{-5}$ \\
$7-3$ & 0.5 & 0.42 & $1.16 \times 10^{-5}$ \\
$3-2$ & 0.5 & 0.42 & $10^{-6}$ \\
$2-1$ & 0.5 & 0.42 & $10^{-6}$ \\
$1-0$ & 0.4 & 0.2 & $3 \times 10^{-6}$ \\
\hline \hline
\end{tabular}

optimization procedure uses the iterative Rabitz algorithm $[78,79]$ and introduces at each step two time propagations allowing the determination of a new field at each time step: an outward one propagating the initial state $\left|\psi_{\text {ini }}(t=0)\right\rangle=$ $|v=56\rangle$ up to $t_{\max }$ followed by a backward one starting from the target $\left|\psi_{\text {target }}\left(t_{\max }\right)\right\rangle=|v=0\rangle$. The performance index is given at each iteration by the overlap between the target and wave packet obtained with the corresponding field. The propagation is carried out in interaction representation without the rotating wave approximation in the basis set of 80 states containing all the vibrational states and the first eigenstates of the trap.

The algorithm converges in two iterations with a performance index of $100 \%$. This indicates the quality of the S-STIRAP field as a guess field for the OCT. The populations driven by the S-STIRAP field and by the optimal field are compared in Fig. 12. The profiles are very similar except at the end to ensure a yield of $100 \%$. Only the levels of the selected chain are drawn for clarity, but the dynamics is performed in the complete basis set constituted by all vibrational levels of the ground state, and the population in the other levels remains negligible. The modulus of the Fourier transform of both fields is compared in Fig. 13, revealing very few modifications by the OCT.

\section{Single optimal chirped pulse}

The last strategy exploits a chirped pulse [80] in order to induce the descent through the successive levels that are coupled by couplings $\mu_{v+1, v}$ presenting a maximum around $v=30$ as illustrated in Fig. 9(b). The energy gap between neighboring levels is varying from 2.14 to $37.3 \mathrm{~cm}^{-1}$. To find a guess field, we use a chirped pulse with constant amplitude $\mathcal{E}(t)=\mathcal{E}_{0} \cos (\omega(t) t)$, where

$$
\omega(t)=\omega_{0}+\alpha t,
$$

$\alpha$ being the chirp rate as defined in Eq. (9).

Finding a relevant parameter set is not easy, and we give here an example providing a yield of $27 \%$ with $\omega_{0}=2 \mathrm{~cm}^{-1}$, $\omega_{f}=20 \mathrm{~cm}^{-1}$, and $t_{\max }=2.41 \mathrm{~ns}$. The rate of frequency increase is $7.5 \mathrm{~cm}^{-1} / \mathrm{ns}$, and this seems reasonable since it does not exceed $10 \%$ per ps. A field amplitude $\mathcal{E}_{0}=4 \times 10^{-5}$ a.u., slightly higher than in the previous strategy, is necessary to get a non-negligible yield. Figure 14 shows the evolution of 

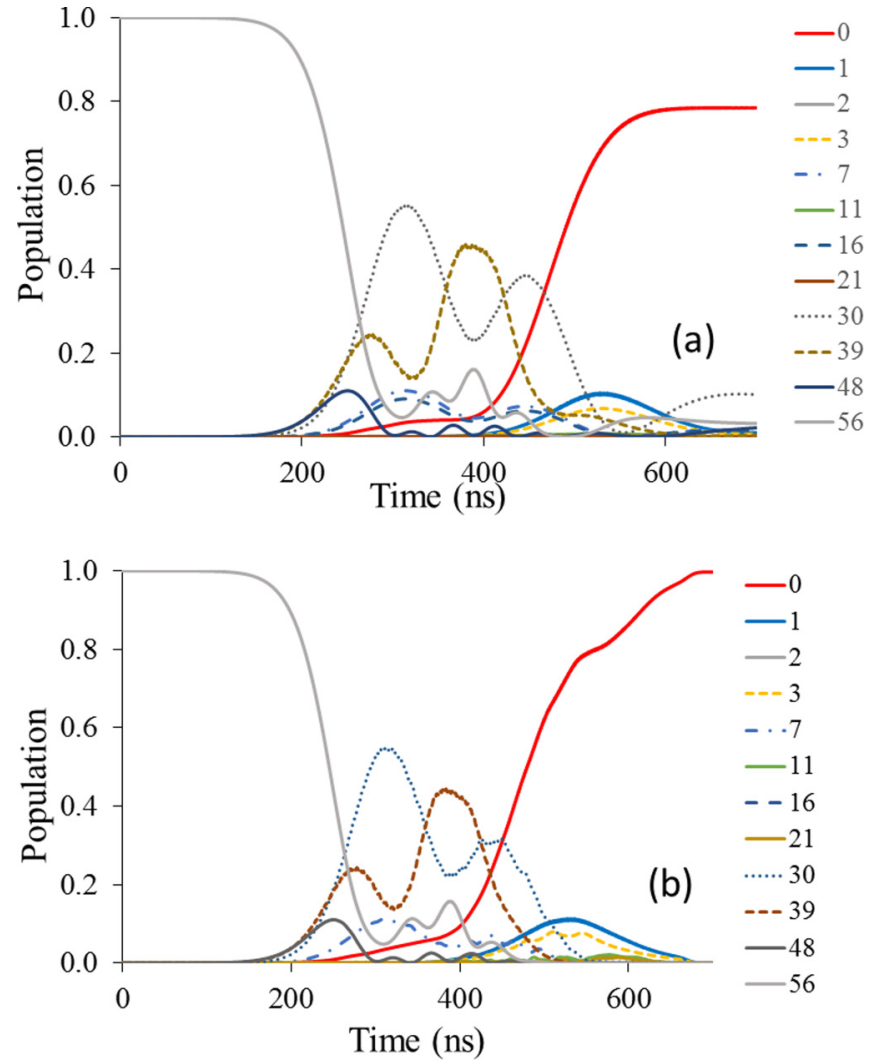

FIG. 12. (a) Population evolution driven by the S-STIRAP sequence with parameters given in Table $\mathrm{V}$ of the Appendix with $t_{\max }=700 \mathrm{~ns}$; (b) evolution with the optimal field when the guess field is this S-STIRAP sequence. Only the levels of the selected chain are drawn for clarity.

the yield with the rate of the chirp for the chosen amplitude and $t_{\max }$. One sees that the yield should strongly decrease if the rate decreases by $3 \%$. On the other hand, increasing the rate does not improve the yield.

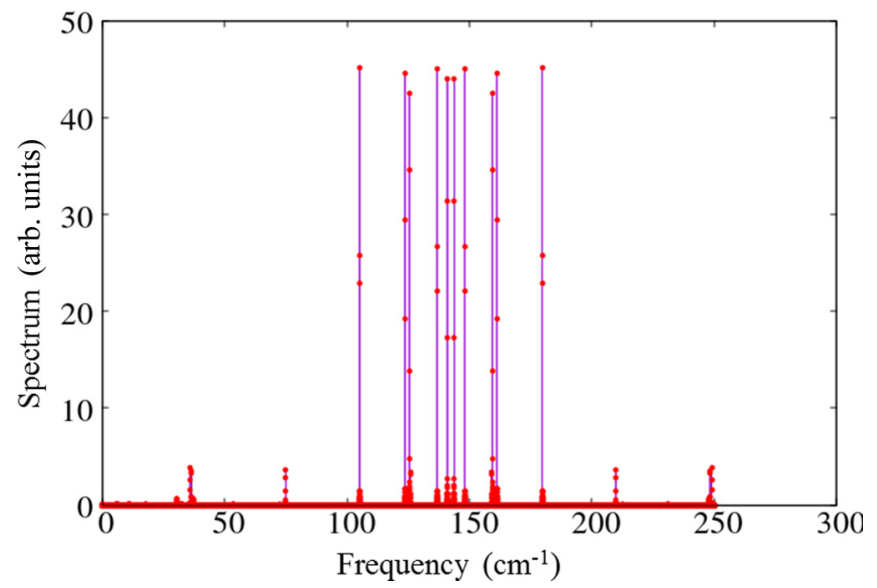

FIG. 13. Modulus of the Fourier transform of guess and OCT fields. Blue full lines: field of the S-STIRAP sequence with parameters given in Table V of the Appendix with $t_{\max }=700 \mathrm{~ns}$; red dots: optimal field when the guess field is this S-STIRAP sequence.

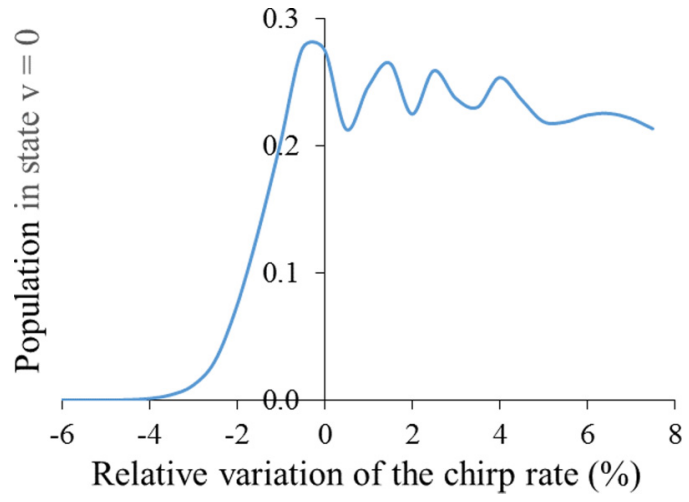

FIG. 14. Variation of the population in the vibrational ground level $v=0$ as a function of the relative variation of the chirp rate [Eq. (14)] with $\omega_{0}=2 \mathrm{~cm}^{-1}, \omega_{f}=20 \mathrm{~cm}^{-1}$, and $t_{\max }=2.41 \mathrm{~ns}$, giving a rate equal to $7.5 \mathrm{~cm}^{-1} / \mathrm{ns}$.

Figure 15(a) shows the population evolution. The early dynamics is complicated, and some levels above $v=56$ are transitory populated. After $0.5 \mathrm{~ns}$, one clearly observes the successive jumps toward the vibrational ground level. As in the previous strategy, we take this chirped pulse as a guess field for OCT. The optimal field again converges in very few iterations by confirming the efficiency of the guess field. Note that a sine square envelope is added in the OCT algorithm to ensure a smooth ramp up and down. The populations are given in Fig. 15(b). The optimal field simplifies the early dynamics. After $0.25 \mathrm{~ns}$, there only remains the regular successive jumps toward the vibrational ground level reached with $100 \%$.
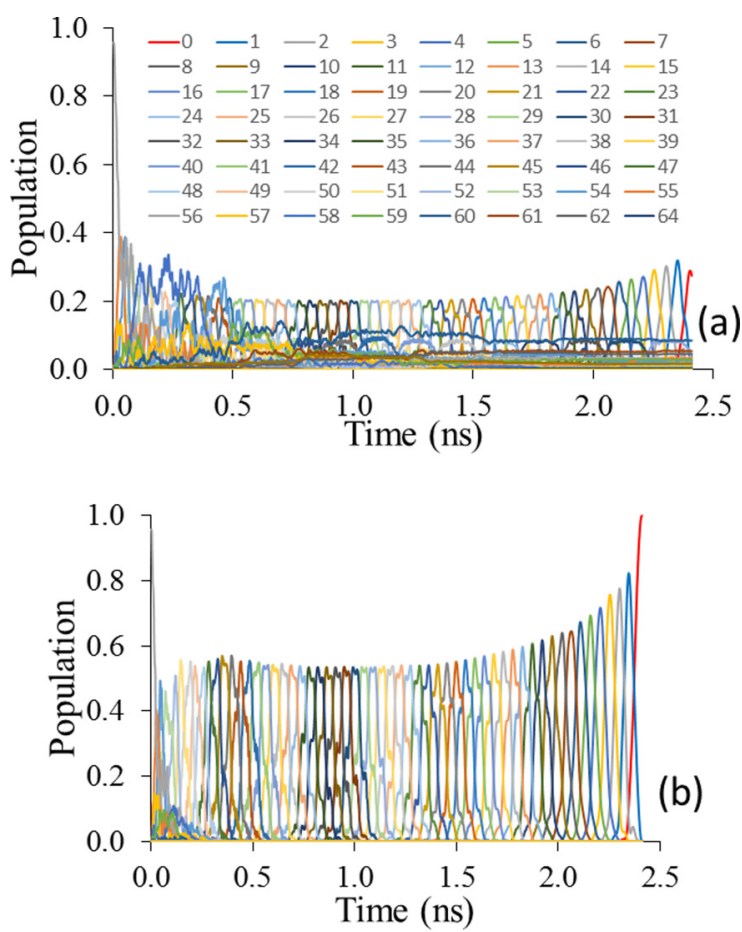

FIG. 15. (a) Population evolution driven by the chirp pulse [Eq. (14)] with parameters $\omega_{0}=2 \mathrm{~cm}^{-1}, \omega_{f}=20 \mathrm{~cm}^{-1}$, and $t_{\max }=$ $2.41 \mathrm{~ns}$; (b) evolution with the optimal field when the guess field is this chirped pulse. 


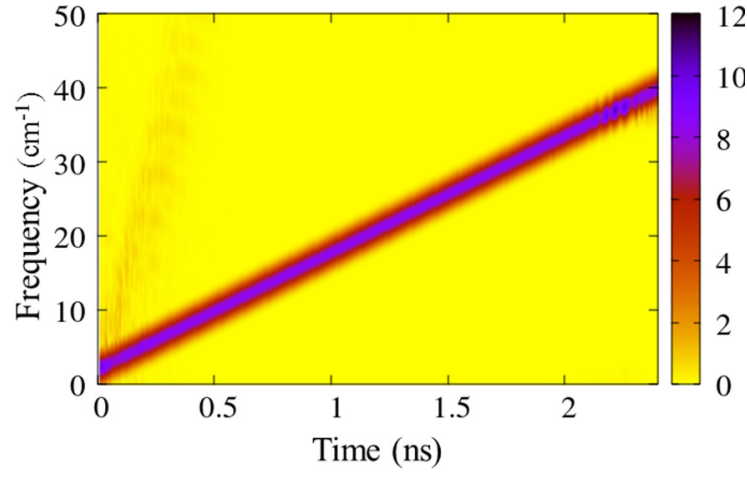

FIG. 16. Spectrogram in arbitrary units of the optimal field using the chirp as a guess field. The chirp parameters are those of Fig. 15.

Figure 16 presents the time-dependent spectrogram of the optimal field obtained by Gabor transform using the Blackman window [81]. As expected from the population evolution shown in Fig. 15, OCT has added some frequencies during the early dynamics before $0.5 \mathrm{~ns}$. The profile has some similarities with a second chirp. The other notable modification is the increase in amplitude at the end of the process. However the maximum amplitude does not exceed $5.8 \times 10^{-5}$ a.u.

\section{CONCLUSION}

In this paper, several full optical strategies with their relative merits and limitations are studied for the formation of $\mathrm{RbSr}$ ultracold molecules in their absolute ground level. Either two electronic states or a single one are involved in the formation dynamics as controlled by linearly polarized laser pulses. Loss of population in intermediate levels, efficiency, and robustness for the molecular bond formation and the subsequent rovibrational transitions down to the rovibrational ground level are the main issues of the processes under consideration. The control schemes refer to two variants of STIRAP, to complete transfer optimized $\pi$-pulses, and to resonant excitation through chirped pulses. The resulting control field characteristics basically involve peak intensities, pulse shape, and duration, in given frequency domains, with the accompanying experimental feasibility criteria, which are compared and discussed.

In summary, the methods, based on STIRAP and involving two electronic states, have the advantage of already being widely used in ultracold investigations. In this context, we show how S-STIRAP is more appropriate than A-STIRAP for reducing the population in the intermediate loosely bound level. The caveat is that stronger fields are required for the $S$ version of STIRAP.

On the other hand, methods involving a single electronic state and referring to IR and $\mathrm{THz}$ pulses do not depend on the properties of excited states, but they must face the challenge of developing $\mathrm{THz}$ laser technology. The formation of a molecular bond with $\mathrm{THz}$ pulses is demonstrated for chirped pulses and $\pi$-pulses. The adiabatic passage is more robust, but the $\pi$-pulse approach requires less intensity. This last point explains that in the near future, $\pi$-pulses are likely to be privileged.

The exploration of the descent through the vibrational levels of the ground electronic state is an attractive perspective since the pulse design in the microwave and far-IR range remains difficult nowadays. However, the simulations on this complex system confirm that such a strategy will be conceivable with simple pulses of moderate intensities inspired from S-STIRAP and chirped pulses. Corrections found by OCT are very weak due to very good guess fields. In the present investigation, we have used an optimization on a time grid, but other methods based on the optimization of the pulse parameters, for instance by genetic algorithms, should provide corrections more directly helpful for pulse shaping.

\section{ACKNOWLEDGMENTS}

This work has been supported in part by the project BLUESHIELD of Agence Nationale de la Recherche (ANR14-CE34-0006), and by the GDR 3575 THEMS of Centre National de la Recherche Scientifique (CNRS). We acknowledge the use of the computing center MésoLUM at Université Paris-Saclay (FR LUMAT 2764). We acknowledge the use of computer resources of Institut de Chimie-Physique.

\section{APPENDIX}

This Appendix displays specific molecule and laser parameters that are referred to when using $\pi$-pulses in the two S-STIRAP sequences illustrated in Figs. 11 and 12 of the main text. Table III gives the frequency, the dipole transition moments, and the width of the Gaussian $\pi$-pulses. Note that the transitions $1 \rightarrow 2$ and $2 \rightarrow 3$ are used only in the STIRAP scheme. The values of the first set of parameters used in the S-STIRAP sequence are gathered in Table IV. The parameters providing a yield of $80 \%$ are given in Table V. They are obtained by systematically varying the parameters of the set given in Table IV.
[1] I. Bloch, Nature (London) 453, 1016 (2008).

[2] A. Micheli, G. K. Brennen, and P. Zoller, Nat. Phys. 2, 341 (2006).

[3] I. M. Georgescu, S. Ashhab, and F. Nori, Rev. Mod. Phys. 86, 153 (2014).

[4] B. Yan, A. Moses, B. Gadway, J. P. Covey, K. R. A. Hazzard, A. M. Rey, D. S. Jin, and J. Ye, Nature (London) 501, 521 (2013).
[5] R. Barnett, D. Petrov, M. Lukin, and E. Demler, Phys. Rev. Lett. 96, 190401 (2006).

[6] K. Shioya, K. Mishima, and K. Yamashita, Mol. Phys. 105, 1283 (2007).

[7] M. Karra, K. Sharma, B. Friedrich, S. Kals, and D. Herschbach, J. Chem. Phys. 144, 094301 (2016).

[8] K. Sharma and B. Friedrich, New J. Phys. 17, 045017 (2015).

[9] D. DeMille, Phys. Rev. Lett. 88, 067901 (2002). 
[10] S. F. Yelin, K. Kirby, and R. Côté, Phys. Rev. A 74, 050301(R) (2006).

[11] A. André, D. DeMille, J. M. Doyle, M. D. Lukin, S. E. Maxwell, P. Rabi, R. J. Schoelkopf, and P. Zoller, Nat. Phys. 2, 636 (2006).

[12] D. Sugny, L. Bomble, T. Ribeyre, O. Dulieu, and M. DesouterLecomte, Phys. Rev. A 80, 042325 (2009).

[13] L. Bomble, P. Pellegrini, P. Ghesquière, and M. DesouterLecomte, Phys. Rev. A 82, 062323 (2010).

[14] A. Jaouadi, E. Barrez, Y. Justum, and M. Desouter-Lecomte, J. Chem. Phys. 139, 014310 (2013).

[15] J. Zhu, S. Kais, Q. Wei, D. Herschbach, and B. Friedrich, J. Chem. Phys. 138, 024104 (2013).

[16] N. Balakrishnan, J. Chem. Phys. 145, 150901 (2016).

[17] C. Makrides, J. Hazra, G. B. Pradhan, A. Petrov, B. K. Kendrick, T. González-Lezana, N. Balakrishnan, and S. Kotochigova, Phys. Rev. A 91, 012708 (2015).

[18] E. R. Hudson, H. J. Lewandowski, B. C. Sawyer, and J. Ye, Phys. Rev. Lett. 96, 143004 (2006).

[19] S. Truppe, R. J. Hendricks, S. K. Tokunaga, H. J. Lewandowski, M. G. Kovlov, C. Henkel, E. A. Hinds, and M. R. Tarbutt, Nat. Commun. 4, 2600 (2013).

[20] J. J. Hudson, D. M. Kara, I. J. Smallman, B. E. Sauer, M. R. Tarbutt, and E. A. Hinds, Nature (London) 473, 493 (2011).

[21] J. Baron, W. C. Campbell, D. DeMille, J. M. Doyle, and G. Gabrielse (ACME Collaboration), Science 343, 269 (2014).

[22] S. K. Tokunaga, C. Stoeffer, F. Auguste, A. Shelkovnikov, C. Daussy, A. Amy-Klein, C. Chardonnet, and B. Darquié, Mol. Phys. 111, 2363 (2013).

[23] E. A. Donley, N. R. Claussen, S. T. Thompson, and C. E. Wieman, Nature (London) 417, 529 (2002).

[24] E. Hodby, S. T. Thompson, C. A. Regal, M. Greiner, A. C. Wilson, D. S. Jin, E. A. Cornell, and C. E. Wieman, Phys. Rev. Lett. 94, 120402 (2005).

[25] E. Timmermans, P. Tommasini, M. Hussein, and A. Kerman, Phys. Rep. 315, 199 (1999).

[26] C. Chin, R. Grimm, P. S. Julienne, and E. Tiesinga, Rev. Mod. Phys. 82, 1225 (2010).

[27] E. Tiesinga, B. J. Verhaar, and H. T. C. Stoof, Phys. Rev. A 47, 4114 (1993).

[28] K. Winkler, F. Lang, G. Thalhammer, P. van der Staten, R. Grimm, and J. Hecker Denschlag, Phys. Rev. Lett. 98, 043201 (2007).

[29] Y. Liu, T. Gong, Z. JI, G. Wang, Y. Zhao, L. Xiao, and S. Jia, J. Chem. Phys. 151, 084303 (2019).

[30] M. Tomza, F. Pawlowski, M. Jeziorska, C. P. Koch, and R. Moszynski, Phys. Chem. Chem. Phys. 13, 18893 (2011).

[31] V. Barbé, A. Ciamei, B. Pasquiou, L. Reichsöllner, F. Schreck, P. S. Żuchowski, and J. M. Hutson, Nat. Phys. 14, 881 (2018).

[32] A. Fioretti, D. Comparat, A. Crubellier, O. Dulieu, F. MasnouSeeuws, and P. Pillet, Phys. Rev. Lett. 80, 4402 (1998).

[33] S. Stellmer, B. Pasquiou, R. Grimm, and F. Schreck, Phys. Rev. Lett. 109, 115302 (2012).

[34] A. Ciamei, A. Bayerle, C.-C. Chen, B. Pasquiou, and F. Schreck, Phys. Rev. A 96, 013406 (2017).

[35] V. S. Malinovsky and D. J. Tannor, Phys. Rev. A 56, 4929 (1997).

[36] I. R. Solá, V. S. Malinovsky, and D. J. Tannor, Phys. Rev. A 60, 3081 (1999).
[37] N. V. Vitanov, A. A. Rangelov, B. W. Shore, and K. Bergmann, Rev. Mod. Phys. 89, 015006 (2017).

[38] S. Kotochigova, Phys. Rev. lett. 99, 073003 (2007).

[39] E. Juarros, P. Pellegrini, K. Kirby, and R. Coté, Phys. Rev. A 73, 041403(R) (2006).

[40] E. Luc-Koenig, R. Kosloff, F. Masnou-Seeuws, and M. Vatasescu, Phys. Rev. A 70, 033414 (2004).

[41] R. Lefebvre and O. Atabek, Phys. Rev. A 101, 063406 (2020).

[42] M. Lewenstein, A. Sanpera, and V. Ahufinger, Ultracold Atoms in Optical Lattices (Oxford University Press, Oxford, 2012).

[43] T. Volz, N. Syassen, D. M. Bauer, E. Hansis, S. Dürr, and G. Rempe, Nat. Phys. 2, 692 (2006).

[44] G. Thalhammer, K. Winkler, F. Lang, S. Schmid, R. Grimm, and J. H. Denschlag, Phys. Rev. Lett. 96, 050402 (2006).

[45] B. Damski, L. Santos, E. Tiemann, M. Lewenstein, S. Kotochigova, P. Julienne, and P. Zoller, Phys. Rev. Lett. 90, 110401 (2003).

[46] T. Stöferle, H. Moritz, K. Gunter, M. Köhl, and T. Esslinger, Phys. Rev. Lett. 96, 030401 (2006).

[47] C. Ospelkaus, S. Ospelkaus, L. Humbert, P. Ernst, K. Sengstock, and K. Bongs, Phys. Rev. Lett. 97, 120402 (2006).

[48] J. K. Freericks, M. M. Maśka, A. Hu, T. M. Hanna, C. J. Williams, P. S. Julienne, and R. Lemanski, Phys. Rev. A 81, 011605(R) (2010).

[49] S. A. Moses, J. P. Covey, M. T. Miecnikowski, B. Yan, B. Gadway, J. Ye, and D. S. Jin, Science 350, 659 (2015).

[50] J. P. Covey, S. A. Moses, M. Gärttner, A. Safavi-Naini, M. T. Miecnikowski, Z. Fu, J. Schachenmayer, P. S. Julienne, A. M. Rey, D. S. Jin, and J. Ye, Nat. Commun. 7, 11279 (2016).

[51] L. Reichsöllner, A. Schindewolf, T. Takekoshi, R. Grimm, and H.-C. Nägerl, Phys. Rev. Lett. 118, 073201 (2017).

[52] N. V. Vitanov, T. Halfmann, B. W. Shore, and K. Bergmann, Annu. Rev. Phys. Chem. 52, 763 (2001).

[53] L. R. Liu, J. D. Hood, Y. Yu, J. T. Zhang, N. R. Hutzler, T. Rosenband, and K. K. Ni, Science 360, 900 (2018).

[54] L. R. Liu, J. D. Hood, Y. Yu, J. T. Zhang, K. Wang, Y.-W. Lin, T. Rosenband, and K. K. Ni, Phys. Rev. X 9, 021039 (2019).

[55] F. Deuretzbacher, K. Plassmeier, D. Pfannkuche, F. Werner, C. Ospelkaus, S. Ospelkaus, K. Sengstock, and K. Bongs, Phys. Rev. A 77, 032726 (2008).

[56] S. Grishkevich and A. Saenz, Phys. Rev. A 76, 022704 (2007).

[57] S. Grishkevich and A. Saenz, Phys. Rev. A 80, 013403 (2009).

[58] S. Grishkevich, S. Sala, and A. Saenz, Phys. Rev. A 84, 062710 (2011).

[59] P. S. Żuchowski, R. Guérout, and O. Dulieu, Phys. Rev. A 90, 012507 (2014).

[60] A. Devolder, E. Luc-Koenig, O. Atabek, M. Desouter-Lecomte, and O. Dulieu, Phys. Rev. A 98, 053411 (2018).

[61] V. Kokoouline, O. Dulieu, R. Kosloff, and F. Masnou-Seeuws, J. Chem. Phys. 110, 9865 (1999).

[62] K. Willner, O. Dulieu, and F. Masnou-Seeuws, J. Chem. Phys. 120, 548 (2004).

[63] S. Kallush and R. Kosloff, Chem. Phys. Lett. 433, 221 (2006).

[64] O. A. Rubtsova, V. I. Kukulin, and V. N. Pomerantsev, Ann. Phys. 360, 613 (2015).

[65] O. Dulieu and P. S. Julienne, J. Chem. Phys. 103, 60 (1995).

[66] O. Dulieu, R. Kosloff, F. Masnou-Seeuws, and G. Pichler, J. Chem. Phys. 107, 10633 (1997).

[67] S. Kallush, R. Kosloff, and F. Masnou-Seeuws, Phys. Rev. A 75, 043404 (2007). 
[68] V. Kokoouline, O. Dulieu, R. Kosloff, and F. Masnou-Seeuws, Phys. Rev. A 62, 032716 (2000).

[69] V. Kokoouline, O. Dulieu, and F. Masnou-Seeuws, Phys. Rev. A 62, 022504 (2000).

[70] P. Pellegrini, O. Dulieu, and F. Masnou-Seeuws, Eur. Phys. J. D 20, 77 (2002).

[71] J. G. Danzl, M. J. Mark, E. Haller, M. Gustavsson, R. Hart, J. Aldegunde, J. M. Hutson, and H.-C. Nägerl, Nat. Phys. 6, 265 (2010).

[72] E. F. de Lima, Phys. Rev. A 95, 013411 (2017).

[73] A. Devolder, E. Luc-Koenig, O. Atabek, M. DesouterLecomte, and O. Dulieu, Phys. Rev. A 100, 052703 (2019).
[74] S. Guérin, V. Hakobyan, and H. R. Jauslin, Phys. Rev. A 84, 013423 (2011).

[75] V. S. Malinovsky and L. Krause, Eur. Phys. J. D 14, 147 (2001).

[76] G. F. Thomas, Phys. Rev. A 27, 2744 (1983).

[77] M. Holthaus and B. Just, Phys. Rev. A 49, 1950 (1994).

[78] C. Brit, R. Chakrabarti, and H. Rabitz, New J. Phys 12, 075008 (2010).

[79] W. Zhu, J. Botina, and H. Rabitz, J. Chem. Phys. 108, 1953 (1998).

[80] S. Chelkowski, A. D. Bandrauk, and P. B. Corkum, Phys. Rev. Lett. 65, 2355 (1990).

[81] M. Sugawara and Y. Fujimura, J. Chem. Phys. 100, 5646 (1994). 